\title{
Une pratique active de communication dans un enseignement de spécialité : la vidéo- correspondance
}

Josiane Hay

\section{(2) OpenEdition}

\section{Journals}

Édition électronique

URL : http://journals.openedition.org/asp/4240

DOI : $10.4000 /$ asp. 4240

ISSN : 2108-6354

\section{Éditeur}

Groupe d'étude et de recherche en anglais de spécialité

Édition imprimée

Date de publication : 1 février 1994

Pagination : $95-100$

ISSN : 1246-8185

\section{Référence électronique}

Josiane Hay, «Une pratique active de communication dans un enseignement de spécialité : la vidéocorrespondance », ASp [En ligne], 3 | 1994, mis en ligne le 21 février 2014, consulté le 19 avril 2019. URL : http://journals.openedition.org/asp/4240 ; DOI : 10.4000/asp.4240

Ce document a été généré automatiquement le 19 avril 2019

Tous droits réservés 


\title{
Une pratique active de communication dans un enseignement de spécialité : la vidéo-correspondance
}

\author{
Josiane Hay
}

1 Dans les enseignements de spécialité, les activités communicatives sont souvent, faute de temps, réduites à la portion congrue. Pour la majorité des ingénieurs et des chercheurs, il est pourtant essentiel de savoir « converser ». La question qui se pose donc à nous tous (et toutes, bien entendu) qui enseignons l'anglais en milieu scientifique, est la suivante: comment développer la compétence communicative; de nos étudiants dans le temps qui nous est imparti et sans réduire notre enseignement de spécialité proprement dit? Les réponses apportées à cette question font généralement intervenir les nouvelles technologies; et le travail individuel; hors des heures de contact avec l'enseignant. La vidéo-correspondance entre dans cette approche. C'est en effet une pratique de communication active qui fait appel à une technologie récente et exige des étudiants de nombreuses heures de travail personnel. C'est en outre une pratique motivante; pour de nombreux étudiants scientifiques, attirés par le caméscope et l'image vidéo. Il m'a donc semblé intéressant d'examiner ici le rôle que peut jouer la vidéo-correspondance dans le développement d'une compétence communicative.

2 Il est difficile de ne pas commencer par une définition de la pratique. Qu'est-ce que la vidéo-correspondance? On est tenté de répondre : l'échange de lettres-vidéo. Mais ceci ne fait que déplacer la question. Comment définir la lettre-vidéo ? Micheline Maurice (Maurice $1991: 37^{1}$ ) détermine celle-ci « comme le résultat, le produit d'un processus de communication, d'échange et de correspondance ». Et nous voyons immédiatement apparaître des notions familières puisque l'objectif principal d'un enseignement de langue est sans conteste de permettre aux apprenants de communiquer, d'échanger dans cette langue. Le problème, nous le savons tous, est de mettre l'apprenant - ici l'étudiant directement en situation, de le faire entrer personnellement dans la langue étrangère. La salle de classe n'est qu'un artifice, et son contexte n'est généralement pas assez 
contraignant. Tout le monde y joue un rôle, mais comme sur une scène, tous les acteurs savent qu'ils sont présence d'autres acteurs et que la réalité est ailleurs. La vidéocorrespondance impose un autre contexte, une autre règle du jeu. La lettre-vidéo est adressée à de vrais «locuteurs natifs", dans mon cas anglophones. Il faudra qu'elle soit comprise. C'est une véritable lettre. Il faudra communiquer quelque chose, et ce quelque chose devra être suffisamment intéressant pour que le destinataire ait envie de répondre. Il ne s'agit donc plus d'un exercice scolaire pour salle de classe, mais d'une communication grandeur nature. Il en résulte que le caméscope se transforme en outil de prise de parole; dans la langue étrangère et les images en chronique interculturelle, et ce sans que l'enseignant ait eu à intervenir puisque, comme dans toute correspondance les auteurs (les deux collectifs) sont responsables du contenu de leur envoi.

Comment ce contexte contraignant se traduit-il, en pratique, dans les lettres-vidéo ? Les séquences que comportent la plupart des lettres-vidéo peuvent être regroupées, du point de vue de leur typologie, sous trois genres principaux :

- la communication personnelle; l'étudiant s'adresse directement à un autre étudiant du groupe destinataire ou à tout le groupe (les salutations font partie de ce genre) ;

- l'interview ;

- le reportage avec commentaire en voix off.

4 Ainsi, l'étudiant qui réalise une lettre-vidéo travaille trois aspects de la compétence communicative. Dans le premier cas, il s'adressera en langue étrangère (ici en anglais) directement à une ou plusieurs personnes pour leur transmettre un message, qui doit avoir un sens. Dans le deuxième cas, il sortira des murs de l'établissement et ira poser des questions en anglais à des personnes qui lui répondront dans la même langue. Pour que l'interview puisse être bien mené, il faudra évidemment que le cameraman et l'étudiant chargé du micro suivent ce qui est dit en anglais. Par la suite, au moment du montage, il faudra que le groupe décide s'il souhaite ou non inclure l'interview dans leur lettre. Pour cela, il faudra que l'ensemble du groupe comprenne le sens des réponses et évalue celuici. Enfin, dans le troisième cas, pour le commentaire en voix off, il s'agit là encore de faire des commentaires à la fois intéressants et compréhensibles. Il faudra donc écrire des textes à plusieurs et les lire en gardant toujours le destinataire à l'esprit. À ces exercices de production s'ajoute l'exercice de compréhension qui fait suite à la réception de la réponse. La vidéo-correspondance constitue donc bien une pratique active de communication. Cependant, l'intérêt du processus d'échange ne s'arrête pas là. La lettrevidéo comporte toujours une dimension interculturelle. Les étudiants de chaque groupe s'exprimeront sur leurs propres réalités, sur leur univers. Ces images de l'autre, envoyées directement par l'autre et non tirées d'une méthode, ont une qualité d'authenticité, qui leur donne une valeur irremplaçable. Cette dimension permet de revitaliser un enseignement de spécialité souvent détaché de toute culture.

5 La vidéo-correspondance n'est pas, bien évidemment, réservée aux étudiants de langue de spécialité. Elle me semble toutefois particulièrement adaptée à ce type de public. D'une part elle permet, ainsi je viens de le montrer, de développer la compétence de communication et d'ajouter une dimension culturelle à l'enseignement de spécialité. D'autre part, comme je l'ai mentionné plus haut, elle motive fortement de nombreux étudiants des disciplines scientifiques ou technologiques qui sont attirés par le caméscope et l'aspect technique du tournage et du montage. Ce goût peut être exploité dans une optique langue de spécialité (Hay 1992): présentation de l'appareil instructions de tournage, etc. en anglais. Par ailleurs, la présentation du cadre de vie et de travail oblige 
les étudiants à acquérir un minimum de lexique de spécialité (chemistry lab, physicist, etc.). La vidéo-correspondance constitue donc bien une pratique active de communication adaptée à un enseignement de spécialité.

\section{BIBLIOGRAPHIE}

Hay. J. 1992. « Vidéo-correspondance et anglais de spécialité ». Les Cahiers de l'APLIUT 12/2, 88-98.

Les carnets du Réseau RVC. 1991. numéro Hors série, juin. CIEP.

Maurice, M. «Réseaux de lettres ». 1991. Les carnets du Réseau RVC numéro Hors série.

Bibliographie complémentaire

Maurice. M. 1987. « Vidéo du réel : troc et communication ». APTE 2, 1819.

RVC Gazette, Revue bi-annuelle du réseau CIEP/Belc de vidéo-correspondance :

RVC Gazette 1. « Le texte du projet. Premières pistes » mai 1983.

RVC Gazette 2. «Premières expériences » mars 1984.

RVC Gazette 3. « Premiers résultats, analyse » janvier 1986.

RVC Gazette 4. « Réseau RVC, réseaux d'images » octobre 1986.

RVC Gazette 5. « Réseaux, troc et pédagogie » printemps 1987.

RVC Gazette 6. « Chronique des lettres-vidéo et pédagogie du projet » mars 1988.

RVC Gazette 7. « La lettre-vidéo à la recherche de ses sources littéraires et cinématographiques » décembre 1988.

RVC Gazette 8. « La vidéo-correspondance, une alchimie pédagogique » mai 1989.

RVC Gazette 9. « La lettre-vidéo, un triple dispositif, pédagogique, vidéographique et interculturel » décembre 1989.

RVC Gazette 10. « Les processus de la correspondance » juin 1990.

RVC Gazette 11. «Carnets : écritures vidéographiques, pratiques pédagogiques, pratiques de formation » mars 1991.

RVC Gazette 12. « Lettres-vidéo, pratiques et réflexion » mai 1992.

Adresse de la messagerie du réseau CIEP/Belc de vidéo-correspondance :

Micheline Maurice, RCVcontacts - CIEP/Belc, 9, rue Lhomond, 75005 Paris.

\section{NOTES}

1. Le Numéro spécial rassemble quelques textes retraçant le cheminement des pratiques et des réflexions qui se mènent dans le réseau vidéo-correspondance depuis presque dix ans. 


\section{RÉSUMÉS}

La communication porte sur une expérience de réalisation de lettres-vidéo par des étudiants de deuxième année de DEUG scientifique (A et B) de l'Université Joseph-Fourier de Grenoble. La présentation de l'expérience est précédée d'une définition de la pratique. Les activités décrites sont placées dans le contexte d'un enseignement de l'anglais de spécialité.

This paper deals with the experimental production of video letters by second-year science students at the University Joseph-Fourier in Grenoble. The definition of the practical aspects are followed by a presentation of the project. The activities described are placed within the framework of teaching ESP.

\section{INDEX}

Mots-clés : anglais de spécialité, communication, vidéo

Keywords : ESP

\section{AUTEUR}

\section{JOSIANE HAY}

Université Joseph-Fourier, Grenoble. josiane.hay@ujf-grenoble.fr 